\title{
Acupuncture Treatment in Patients with Low Back Pain
}

\author{
Jihe Zhu1, Blagica Arsovska ${ }^{1,2}$, Kristina Kozovska1 ${ }^{*}$ \\ ${ }^{1}$ Faculty of Medical Sciences, Goce Delchev University, Shtip, Republic of Macedonia \\ ${ }^{2}$ Institute of Biology, Faculty of Natural Sciences and Mathematics, Skopje, Republic of Macedonia \\ Email: *tongdatang-tcm@hotmail.com
}

How to cite this paper: Zhu, J.H., Arsovska, B. and Kozovska, K. (2018) Acupuncture Treatment in Patients with Low Back Pain. Yangtze Medicine, 2, 203-207. https://doi.org/10.4236/ym.2018.24022

Received: July 27, 2018

Accepted: August 27, 2018

Published: August 30, 2018

Copyright $\odot 2018$ by authors and Scientific Research Publishing Inc. This work is licensed under the Creative Commons Attribution International License (CC BY 4.0).

http://creativecommons.org/licenses/by/4.0/ (c) (i) Open Access

\begin{abstract}
Low back pain is the most common medical problem and very commonly treated condition with acupuncture today. The pain can arise from muscles, tendons, ligaments, bones or intervertebral discs. The pain is usually in the lower back region, gluteal region with or without radiation in the legs, with muscle tension and stiffness, limited movement or sometimes tingling and burning sensation. In the term of Traditional Chinese Medicine (TCM), pain appears if there is no good Blood and Qi flow in the body. Pain caused by Qi stagnation usually appears due to strong emotional and mental changes and stress. In this research, we included 60 patients, 28 males and 32 females, on age from 18 to 88, all treated with acupuncture for low back pain. All patients were cured with certain number of treatments. Most of the patients needed less than 5 treatments. Treatments were made with normal and fire needle and combined. The best results were achieved with fire needle acupuncture treatment. In the treatment, we used local Ashi i.e. trigger points and meridian points: BL24 (QiHaiSu), BL25 (DaChangShu), BL26 (GuanYanShu), BL27 (XioChangShu), BL28 (PangGuangShu), GB30 (HuanTiao), BL40 (WeiZhong), BL57 (ChengShen), GB37 (GuanMing) and BL60 (KunKun). Acupuncture as a treatment for low back pain is a very effective treatment giving very satisfying and positive results in a short time of period. The percentage of cured patients (relieved of the symptoms) is $100 \%$ which is a clear indicator of the success of acupuncture in the treatment of low back pain.
\end{abstract}

\section{Keywords}

Traditional Chinese Medicine, Acupuncture, Treatment, Lumbar, Pain

\section{Introduction}

Low back pain is the most common medical problem and very commonly 
treated condition with acupuncture today. The pain can arise from muscles, tendons, ligaments, bones or intervertebral discs. The pain is usually in the lower back region, gluteal region with or without radiation in the legs, with muscle tension and stiffness, limited movement or sometimes tingling and burning sensation [1]. Low back pain affects both women and men and increases in the aging population. The pain can be also influenced by the psychological factors: anxiety, stress and depression [2]. Low back pain can be defined as:

1) Acute pain - present pain for less than 6 weeks;

2) Subacute pain-present pain between 6 weeks and 3 months;

3) Chronic pain-present pain for longer than 3 months [1].

In the term of Traditional Chinese Medicine (TCM), pain appears if there is no good Blood and Qi flow in the body. Pain caused by Qi stagnation usually appears due to strong emotional and mental changes and stress. If there is Blood stasis, there will be present sharp pain at a specific location and painful swelling. If there is insufficiency of Qi and Blood, the pain is not severe but it lasts longer and becomes worse after rest. The pain is worse after rest, because there is insufficient Blood and Qi to keep the flow moving. Movement helps to improve the Qi and Blood flow. Before starting the treatment, the practitioner must know if the pain is due to insufficiency of blockage, Blood stasis or Qi stagnation, what is the cause and the meridians that are affected. Therefore, every patient in TCM is given an individual treatment based on the pattern of disharmony.

The Qi and the Blood flow can be affected by the external pathogenic factors: wind, cold, dampness, heat and etc. and when they invade the low back, they cause low back pain. If they are present in the upper body, cold or flu appears. Low back pain caused by wind is very achy, and caused by cold, the pain is located in one point, very sharp and severe. Cold is also causing Blood stasis. Pain caused by dampness is fixed, but very heavy and usually chronic. If there is swelling, it results from too much fluid accumulation. Heat causes swelling, redness and inflammation, especially in the joints. A person also must have strong Liver and Kidney, so the invasion of the external pathogens will be short and acute.

Internal factors that can cause low back pain are the emotions. Liver depression caused by frustration and stress can cause sciatica pain. Kidney can be damaged by constant fear and make the low back area weak. Worry and overthinking can damage the spleen and cause Blood insufficiency [3].

The principle treatment in TCM is to get the Blood and Qi flow free as soon as possible, remove the blockages from the Blood, expel the exogenous pathogenic factors, warm and invigorate the Blood, eliminate the inflammation and improve the function of whole body [4].

\section{Material and Methods}

In this research are included 60 patients, 28 males and 32 females, on age from 18 to 88 , all treated with acupuncture for low back pain.

The patients were randomly selected from the archive of the clinic treated for low back pain in a period of three years 2015, 2016 and 2017. The number of pa- 
tients, age and sex were randomly selected. Patients are mostly from a city urban area with a sedentary lifestyle.

Inclusion criteria. In the treatment are included patients who have finished the treatments, patients with low back pain with or without radiation of the pain in the leg, patients with hip pain, patients with acute and chronic pain, patients with osteoporosis, trauma injury, discus hernia, sciatica pain, muscle sprain, degenerative diseases, spondylolysis, spondylitis and vertebral spondylolisthesis.

Exclusion criteria. In the treatment were not included patients who haven't finished the treatments, cancer patients, pregnant patients, children and patients with localized pain on other parts of the body.

Ethical clearance of the study was claimed from all the patients before starting the treatment.

The patients were treated with acupuncture treatment in a clinic for TCM and acupuncture in Skopje, Macedonia by a doctor specialist in acupuncture. Treatments were made with normal and fire needle acupuncture and combined. Treatments with normal needle were with duration of 35 - 40 minutes on Ashi and meridian points. Treatments with fire needle were with duration of 5 - 10 minutes on local Ashi points. Treatments were done indoor, on a room temperature, once a week, ten treatments in one session. In the treatment were used fine sterile disposable needles $0.25 \times 25 \mathrm{~mm}$ manufactured by Wuijuiang City Medical \& Health Material Co., LTD.

In the treatment were used local Ashi i.e. trigger points and meridian points: BL24 (QiHaiSu), BL25 (DaChangShu), BL26 (GuanYanShu), BL27 (XioChangShu), BL28 (PangGuangShu), GB30 (HuanTiao), BL40 (WeiZhong), BL57 (ChengShen), GB37 (GuanMing) and BL60 (KunKun).

\section{Results and Discussion}

After treatments, patients were monitored and were coming for doctoral examinations. During controls, patients explained how they feel after treatments, whether there is more pain and other accompanying symptoms. All patients were cured (relieved of the symptoms) from the low back pain with certain number of treatments and recurrence of pain was not reported by any patient, although it is possible to happen in some cases. If there is pain recurrence, patients are encouraged to report it always and come for more treatments.

$47 \%$ or 28 patients needed less than 5 treatments to achieve the results. In $\mathrm{Ta}$ ble 1 it is shown the number of patients and treatments made. $33 \%$ or 20 patients needed 5 to 10 treatments, 9 patients (15\%) needed 10 to 15 treatments and only 3 patients (5\%) needed more than 15 treatments.

The best results were achieved with fire needle acupuncture treatment. 48 patients were treated with fire needle, 5 patients with normal needle and 7 patients combined with fire and normal needle. The same results are shown in Table 2. In our practice the result are showing that the treatment with fire needle gives better results that the treatment with normal needle in conditions like low back pain, knee pain, elbow and other. 
Of the treated patients, 32 were female and 28 male, but these numbers are irrelevant. The patients were on age from 18 to 88 . Patients were divided in 7 age groups. Most of the patients (27\%, 16 patients) were older than 70 . The results for age groups are shown in Table 3.

30 of the treated patient were also taking pain medications occasionally. 10 of the patients had insomnia, and 5 patients had constipation. Of the other symptoms were present those typical for low back pain syndrome: radiating pain in the knees, legs and hips, restricted movement, pain when lifting heavy objects, numbness, tingling, burning sensation, edema, stiff muscles and etc. Most patients had pain in the level of L4/L5 and L5/S1.

The duration of the pain was different in all patients. Some have the pain for 15 - 20 years, for 1 - 2 years, 3 months, 2 weeks or few days. 37 patients had chronic pain and 23 had acute pain. Patients with acute pain were treated with normal needle acupuncture and combined treatment. Patients treated for chronic pain needed in average 7 treatments. Patients treated for acute pain needed in average 5 treatments.

The treated acupoints were chosen with aim to help the patients to eliminate the pain, remove the accompanying symptoms, to restore the function of the

Table 1. Number of treatments made.

\begin{tabular}{cc}
\hline Number of treatments & Number of patients \\
\hline$<5$ & $28(47 \%)$ \\
$5-10$ & $20(33 \%)$ \\
$10-15$ & $9(15 \%)$ \\
$>15$ & $3(5 \%)$ \\
\hline
\end{tabular}

Table 2. Number of patients who used the different type of acupuncture treatment.

\begin{tabular}{cc}
\hline Type of acupuncture treatment & Number of patients \\
\hline Fire needle & $48(80 \%)$ \\
Normal needle & $5(8 \%)$ \\
Combined & $7(12 \%)$ \\
\hline
\end{tabular}

Table 3. Age groups.

\begin{tabular}{cc}
\hline Age group & Number of patients \\
\hline$<20$ & $1(2 \%)$ \\
$20-30$ & $2(3 \%)$ \\
$30-40$ & $8(13 \%)$ \\
$40-50$ & $11(18 \%)$ \\
$50-60$ & $9(15 \%)$ \\
$60-70$ & $13(22 \%)$ \\
$>70$ & $16(27 \%)$ \\
\hline
\end{tabular}


muscles, tendons, ligaments, joints and bones, to ensure free and painless movement of the body and remove all the blockages from the Blood and Qi flow. The points are located on the upper back, middle and lower back, hips and legs [5].

Acupuncture benefits all parts of the body. Acupuncture can significantly reduce the pain and inflammation in the affected area, reduce the disability, improve the function, reduce the functional limitations and correct the Blood and Qi flow in the affected area [4] [6] [7].

\section{Conclusion}

Acupuncture as a treatment for low back pain is a very effective treatment giving very satisfying and positive results in a short time of period. The percentage of cured patients (relieved of the symptoms) is $100 \%$ which is a clear indicator of the success of acupuncture in the treatment of low back pain.

\section{Conflicts of Interest}

The authors declare that there are not conflicts of interest.

\section{References}

[1] Koes, B.W., van Tulder, M.W. and Thomas, S. (2006) Diagnosis and Treatment of Low Back Pain. BMJ, 332, 1430-1434. https://doi.org/10.1136/bmj.332.7555.1430

[2] Allegri, M., et al. (2016) Mechanisms of Low Back Pain: A Guide for Diagnosis and Therapy. Version 2, F1000Res., F1000 Faculty Rev-1530.

https://doi.org/10.12688/f1000research.8105.2

[3] Douglas, F. (1995) Low Back Pain: Care \& Prevention with Traditional Chinese Medicine. Blue Poppy Pr, 1st Edition, ISBN 0-936185-66-X.

[4] Ilieva, V., Zhu, J., Arsovska, B. and Kozovska, K. (2018) Acupuncture Treatment in Patients with Lumbar Degenerative Disc Disease and Hip Pain. Imperial Journal of Interdisciplinary Research (IJIR), 4, No. 1.

[5] White, A. (2017) Does Acupuncture Help Back Pain? http://www.healthline.com/

[6] Zanardi, P. (2004) Acupuncture Considerations. http://www.healthline.com/

[7] Liu, L., et al. (2015) Acupuncture for Low Back Pain: An Overview of Systematic Reviews. Evidence-Based Complementary and Alternative Medicine, 2015, Article ID: 328196. 